\title{
Ground states for the SOS model with an external field on the Cayley tree M.M.Rahmatullaev, M.R.Abdusalomova, M.A.Rasulova
}

\begin{abstract}
We consider a nearest-neighbor solid-on-solid (SOS) model, with several spin values $0,1,2, \ldots, m, m \geq 2$ and non zero external field, on a Cayley tree of order $k$. In the case $k=2, m=2$, we describe translation-invariant ground states for the SOS model with a translation-invariant external field. Some periodic ground states for the SOS model with periodic external field are described.

Keywords: Cayley tree, SOS model, external field, translation-invariant external field, periodic external field, configuration, translation-invariant ground state, periodic ground state.
\end{abstract}

\section{Introduction}

One of fundamental problems is to describe the extreme Gibbs measures corresponding to a given Hamiltonian. Each Gibbs measure is associated with a single phase of a physical system. Existence of two or more Gibbs measures means that phase transitions exist.

As is known, the phase diagram of Gibbs measures for a Hamiltonian is close to the phase diagram of isolated (stable) ground states of this Hamiltonian. At low temperatures, a periodic ground state corresponds to a periodic Gibbs measure.Therefore the problem of description of periodic ground states naturally arises (see [1], [3],[5]-[9]).

We consider a nearest-neighbor solid-on-solid (SOS) model, with several spin values $0,1,2, \ldots, m, m \geq$ 2 and non zero external field, on a Cayley tree of order $k$. The SOS model can be treated as a natural generalization of the Ising model (obtained for $m=1$ ). We mainly assume that $m=2$ (three spin values) and study translation-invariant and periodic ground states.

In [5] and [6],[8] for the Ising model with competing interactions, periodic and weakly periodic ground states were described.

In [7] for the Potts model with competing interactions on the Cayley tree of order $k$ with $k \geq 2$, periodic and weakly periodic ground states for normal subgroups of index 4 were studied.

In [3] for the $\lambda$-model on the Cayley tree of order two, periodic and weakly periodic ground states were studied.

In [9] for the Ising model on the Cayley tree of order two, translation-invariant, periodic ground states were described.

In this paper we shall study translation-invariant and periodic ground states for the SOS model with external fields.

\section{Main definitions and known facts}

Let $\tau^{k}=(V, L)$ be a Cayley tree of order $k$, i.e, an infinite tree such that exactly $k+1$ edges are incident to each vertex. Here $V$ is the set of vertices and $L$ is the set of edges of $\tau^{k}$.

Let $G_{k}$ denote the free product of $k+1$ cyclic groups $\left\{e, a_{i}\right\}$ of order 2 with generators $a_{1}, a_{2}, \ldots, a_{k+1}$, i.e., let $a_{i}^{2}=e$ (see [4]).

There exists a one-to-one correspondence between the set $V$ of vertices of the Cayley tree of order $k$ and the group $G_{k}$, (see [1],[2]).

We show how to construct this correspondence. We choose an arbitrary vertex $x_{0} \in V$ and associate it with the identity element $e$ of the group $G_{k}$. Since we may assume that the graph under consideration is planar, we associate each neighbor of $x_{0}$ (i.e., $e$ ) with a single generator $a_{i}, i=1,2, \ldots, k+1$, where the order corresponds to the positive direction, see Figure 1. 


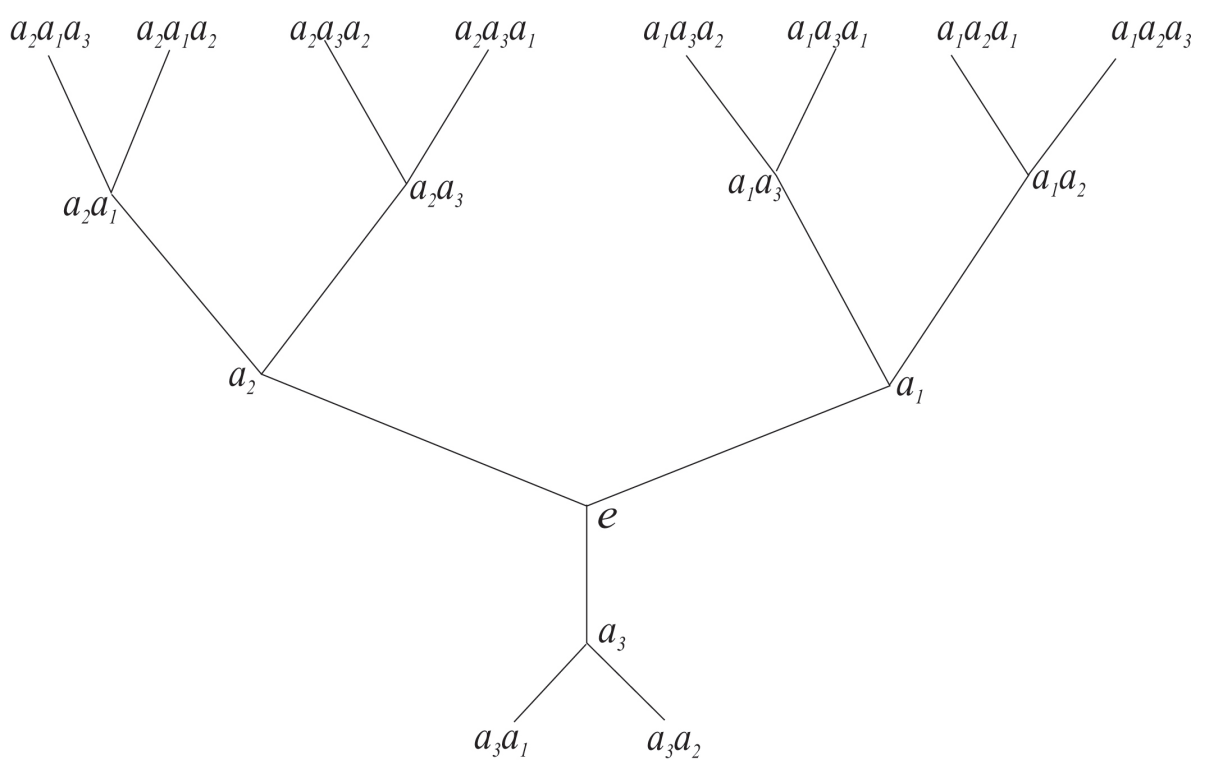

Figure 1: The Cayley tree $\tau^{2}$ and elements of the group representation of vertices

For every neighbor of $a_{i}$, we introduce words of the form $a_{i} a_{j}$. Since one of the neighbors of $a_{i}$ is $e$, we put $a_{i} a_{i}=e$. The remaining neighbors of $a_{i}$ are labeled according to the above order. For every neighbor of $a_{i} a_{j}$, we introduce words of length 3 in a similar way. Since one of the neighbors of $a_{i} a_{j}$ is $a_{i}$, we put $a_{i} a_{j} a_{j}=a_{i}$. The remaining neighbors of $a_{i} a_{j}$ are labeled by words of the form $a_{i} a_{j} a_{l}$, where $i, j, l=1,2, \ldots, k+1$, according to the above procedure. This agrees with the previous stage because $a_{i} a_{j} a_{j}=a_{i} a_{j}^{2}=a_{i}$. Continuing this process, we obtain a one-to-one correspondence between the vertex set of the Cayley tree $\tau^{k}$ and the group $G_{k}$.

The representation constructed above is said to be right because, for all adjacent vertices $x$ and $y$ and the corresponding elements $g, h \in G_{k}$, we have either $g=h a_{i}$ or $h=g a_{j}$ for suitable $i$ and $j$. The definition of the left representation is similar.

For the group $G_{k}$ (or the corresponding Cayley tree), we consider the left (right) shifts. For $g \in G_{k}$, we put

$$
T_{g}(h)=g h\left(T_{g}(h)=h g\right) \text { for all } h \in G^{k}
$$

The group of all left (right) shifts on $G_{k}$ is isomorphic to the group $G_{k}$.

Each transformation $S$ on the group $G_{k}$ induces a transformation $S$ on the vertex set $V$ of the Cayley tree $\tau^{k}$. In the sequel, we identify $V$ with $G_{k}$.

The following assertion is quite obvious (see [1], 2]).

Theorem 2.1. The group of left (right) shifts on the right (left) representation of the Cayley tree is the group of translations.

For each $x \in G_{k}$, let $S_{1}(x)$ denote the set of all neighbors of $x$, i.e., $S_{1}(x)=\left\{y \in G_{k}:\langle x, y\rangle\right\}$, where $\langle x, y\rangle$ means that the vertex $x$ and $y$ are nearest neighbor.

Assume that spin takes its values in the set $\Phi=\{0,1,2, \ldots, m\}$. By a configuration $\sigma$ on $V$ we mean a function taking $\sigma: x \in V \rightarrow \sigma(x) \in \Phi$. The set of all configurations coincides with the set $\Omega=\Phi^{V}$.

Consider the quotient group $G_{k} / G_{k}^{*}=\left\{H_{1}, \ldots, H_{r}\right\}$, where $G_{k}^{*}$ is a normal subgroup of index $r$ with $r \geq 1$. 
Definition 2.1. A configuration $\sigma(x)$ is said to be $G_{k}^{*}$-periodic if $\sigma(x)=\sigma_{i}$ for all $x \in G_{k}$ with $x \in H_{i} . A G_{k}-$ periodic configuration is said to be translation invariant.

By period of a periodic configuration we mean the index of the corresponding normal subgroup.

SOS model with an external field is given by Hamiltonian:

$$
H(\sigma)=-J \sum_{\langle x, y\rangle \in L}|\sigma(x)-\sigma(y)|+\sum_{x \in V} \alpha_{x} \sigma(x),
$$

where $J \in \mathbb{R}, \alpha_{x}$ is an external field and $\sigma \in \Omega$. As usual, $\langle x, y\rangle$ stands for nearest neighbour vertices.

The SOS model of this type can be considered as a generalization of the Ising model (which arises when $m=1$ ). Here, $J<0$ gives a ferromagnetic (FM) and $J>0$ an antiferromagnetic (AFM) model. In the FM case with zero external field the ground states are "flat" configurations, with $\sigma(x)=j \in \Phi$ (there are $m+1$ of them), in the AFM two "contrasting" checher-board configurations, where $|\sigma(x)-\sigma(y)|=m$, $\forall<x, y>$.

Comparing with the Potts model (see, e.g., Refs. 10-14), the SOS with zero external field has "less symmetry" and therefore more diverse structure of phases. For example, in the FM case it is intuitively plansible that the ground states corresponding to "middle-level" surfaces will be "dominant". This observation was made formal in Ref's for the model on a cubic lattice.

\section{Model with an external field}

Let $M$ be the set of all unit balls with vertices in $V$, i.e.

$$
M=\left\{\{x\} \bigcup S_{1}(x): \forall x \in V\right\} .
$$

By the restricted configuration $\sigma_{b}$ we mean the restriction of a configuration $\sigma$ to a ball $b \in M$. Let $c_{b}$ denote the center of a unit ball $b$. The energy of a configuration $\sigma_{b}$ on $b$ is defined by the formula

$$
U\left(\sigma_{b}\right)=-\frac{1}{2} J \sum_{\substack{\langle x, y\rangle \\ x, y \in b}}|\sigma(x)-\sigma(y)|+\alpha_{c_{b}} \sigma\left(c_{b}\right) .
$$

Note that $U\left(\sigma_{b}\right)$ has finitely many values for arbitrary configuration $\sigma$.

Definition 3.1. A configuration $\varphi$ is called a ground state for the Hamiltonian 2.1, if

$$
U\left(\varphi_{b}\right)=\min \left\{U_{\psi_{b}}\right\}
$$

for any $\psi \in \Omega$ and $b \in M$.

Theorem 3.1. Let $k=2, m=2$. For the SOS model with arbitrary non-zero external field, if a translation-invariant configuration is a ground state, then the external field is translation-invariant.

Proof. We shall prove that if translation-invariant configuration $\sigma(x)=2, \forall x \in V$ is a ground state, then the external field is translation-invariant.

Assume, $\alpha_{x} \in\left\{\alpha_{0}, \alpha_{1}, \ldots, \alpha_{n}, \ldots\right\}, \forall x \in V$.

Let $\sigma(x)=2, \forall x \in V$ be a ground state. Then the energy of the unit balls $b \in M$ may be one of the following:

$$
2 \alpha_{0}, 2 \alpha_{1}, 2 \alpha_{2}, \ldots, 2 \alpha_{n}, \ldots
$$

Since $\sigma(x)=2, \forall x \in V$ is a ground state the energy $2 \alpha_{0}$ must be minimal. From minimality of this energy for variable of external field we get the following set $\left\{\left(\alpha_{0}, \alpha_{1}, \ldots, \alpha_{n}\right): 2 \alpha_{0} \leq 2 \alpha_{1}, \ldots, 2 \alpha_{0} \leq\right.$ 
$\left.2 \alpha_{n}, \ldots\right\}$. From minimality of $2 \alpha_{1}$ we take the set $\left\{\left(\alpha_{0}, \alpha_{1}, \ldots, \alpha_{n}\right): 2 \alpha_{1} \leq 2 \alpha_{0}, 2 \alpha_{1} \leq 2 \alpha_{2}, \ldots, 2 \alpha_{1} \leq\right.$ $\left.2 \alpha_{n}, \ldots\right\}$ etc. Consequently we take the following:

$$
\begin{gathered}
\left\{\left(\alpha_{0}, \alpha_{1}, \ldots, \alpha_{n}, \ldots\right): \alpha_{0} \leq \alpha_{1}, \ldots, \alpha_{0} \leq \alpha_{n}, \ldots\right\} \cap \\
\cap\left\{\left(\alpha_{0}, \alpha_{1}, \ldots, \alpha_{n}, \ldots\right): \alpha_{1} \leq \alpha_{0}, \alpha_{1} \leq \alpha_{2}, \ldots, \alpha_{1} \leq \alpha_{n}, \ldots\right\} \cap \ldots \\
\cap\left\{\left(\alpha_{0}, \alpha_{1}, \ldots, \alpha_{n}, \ldots\right): \alpha_{n} \leq \alpha_{0}, \alpha_{n} \leq \alpha_{1}, \ldots\right\} \equiv \\
\equiv\left\{\left(\alpha_{0}, \alpha_{1}, \ldots, \alpha_{n}, \ldots\right): \alpha_{0}=\alpha_{1}=\ldots=\alpha_{n} \ldots\right\},
\end{gathered}
$$

i.e. external field must be a translation-invariant.

\section{This finishes the proof of Theorem 3.1.}

Remark 3.1. Note that configuration $\sigma(x)=0, \forall x \in V$ is a translation-invariant, but it may be ground states for any $\alpha \geq 0$ and the configuration $\sigma(x)=1, \forall x \in V$ is a ground state, if the external field is equal to zero.

SOS model with a translation-invariant external field, i.e. $\alpha_{x}=\alpha, \forall x \in V$, is defined by the following Hamiltonian:

$$
H(\sigma)=-J \sum_{\langle x, y\rangle \in L}|\sigma(x)-\sigma(y)|+\alpha \sum_{x \in V} \sigma(x),
$$

were $J, \alpha \in \mathbb{R}$. The energy of configuration $\sigma_{b}$ on $b$ is defined by the formula

$$
U\left(\sigma_{b}\right)=-\frac{1}{2} J \sum_{\substack{\langle x, y\rangle \\ x, y \in b}}|\sigma(x)-\sigma(y)|+\alpha \sigma\left(c_{b}\right) .
$$

It is not difficult to prove the following Lemma.

Lemma 3.1. Let $k=2$. For each configuration $\sigma_{b}$, we have the following

$$
U\left(\sigma_{b}\right) \in\left\{U_{1}\left(\sigma_{b}\right), \ldots, U_{18}\left(\sigma_{b}\right)\right\},
$$

where

$$
\begin{array}{ccc}
U_{1}\left(\sigma_{b}\right)=-\frac{J}{2}, & U_{2}\left(\sigma_{b}\right)=-J, & U_{3}\left(\sigma_{b}\right)=-\frac{3 J}{2}, \\
U_{4}\left(\sigma_{b}\right)=-2 J, & U_{5}\left(\sigma_{b}\right)=-\frac{5 J}{2}, & U_{6}\left(\sigma_{b}\right)=-3 J, \\
U_{7}\left(\sigma_{b}\right)=-\frac{J}{2}+\alpha, & U_{8}\left(\sigma_{b}\right)=-J+\alpha, & U_{9}\left(\sigma_{b}\right)=-\frac{3 J}{2}+\alpha, \\
U_{10}\left(\sigma_{b}\right)=-\frac{J}{2}+2 \alpha, & U_{11}\left(\sigma_{b}\right)=-J+2 \alpha, & U_{12}\left(\sigma_{b}\right)=-\frac{3 J}{2}+2 \alpha, \\
U_{13}\left(\sigma_{b}\right)=-2 J+2 \alpha, & U_{14}\left(\sigma_{b}\right)=-\frac{5 J}{2}+2 \alpha, & U_{15}\left(\sigma_{b}\right)=-3 J+2 \alpha, \\
U_{16}\left(\sigma_{b}\right)=\alpha, & U_{17}\left(\sigma_{b}\right)=2 \alpha, & U_{18}\left(\sigma_{b}\right)=0 .
\end{array}
$$

For every $i=\overline{1,18}$, we put $A_{i}=\left\{(J, \alpha): U_{i}\left(\sigma_{b}\right) \leq U_{j}, j=\overline{1,18}\right\}$.

Quite combersome but not difficult calculations show that:

$A_{1}=A_{2}=A_{3}=A_{4}=A_{5}=\{(J, \alpha): J=0, \alpha \geq 0\}$,

$A_{6}=\{(J, \alpha): J \geq 0, \alpha \geq 0\}, \quad A_{7}=A_{8}=A_{9}=\{(J, \alpha): J=0, \alpha=0\}$,

$A_{10}=A_{11}=A_{12}=A_{13}=A_{14}=\{(J, \alpha): J=0, \alpha \leq 0\}$,

$A_{15}=\{(J, \alpha): J \geq 0, \alpha \leq 0\}, \quad A_{16}=\{(J, \alpha): J \leq 0, \alpha=0\}$,

$A_{17}=\{(J, \alpha): J \leq 0, \alpha \leq 0\}, \quad A_{18}=\{(J, \alpha): J \leq 0, \alpha \geq 0\}$.

The following theorem describes the necessary condition for a configuration to be a ground state for the SOS model with arbitrary non-zero external fields.

Theorem 3.3. For the SOS model with arbitrary non-zero external field, if the external field is translation-invariant, then arbitrary ground state is translation-invariant.

Proof. Let $\sigma$ be arbitrary ground state. For any $b \in M$ we consider the following sets: 


$$
\Omega_{b, i}=\left\{\sigma_{b}: \sigma_{b}\left(c_{b}\right)=i\right\}, i=0,1,2
$$

May be the following cases:

1) Let at least two sets $\Omega_{b, i}, i=0,1,2$ are nonempty:

a) If the sets $\Omega_{b, 2}$ and $\Omega_{b, 3}$ are nonempty, then $\sigma$ configuration is no translation-invariant and it is ground state on the set $A_{i} \bigcap A_{j}=\{(J, \alpha): \alpha=0\}$,

$i \in\{7,8,9,16\}, j \in\{10,11,12,13,14,15,17\}$, i.e external field must equal to zero.

b) If the sets $\Omega_{b, 1}$ and $\Omega_{b, 2}$ are nonempty, then $\sigma$ configuration is no translation-invariant and it is ground state on the set $A_{i} \bigcap A_{j}=\{(J, \alpha): \alpha=0\}, i \in\{1,2,3,4,5,6,18\}, \quad j \in\{7,8,9,16\}$, i.e external field must equal to zero.

c) If sets $\Omega_{b, 1}$ and $\Omega_{b, 3}$ are nonempty, then $\sigma$ configuration is no translation-invariant and it is ground state on the set $A_{i} \bigcap A_{j}=\{(J, \alpha): \alpha=0\}, i \in\{1,2,3,4,5,6,18\}, j \in\{10,11,12,13,14,15,17\}$, i.e external field must equal to zero.

2) If only one of the sets is nonempty and another two sets is empty, then $\sigma$ is translation-invariant.

This finishes the proof of Theorem 3.3.

Remark 3.2. In [9] Ising model with non-zero external field is considered and a necessary and sufficiency conditions for the external field are found to make a translation-invariant configuration a ground state. Note that for model of SOS such necessary and sufficiency conditions a not known.

We let $G S(H)$ denote the set of all ground states of the Hamiltonian $H$ (see (3.2)).

Theorem 3.4. For the SOS model with non-zero translation-invariant external field (i.e. for the Hamiltonian (3.2)).

a) If $(J, \alpha) \in A_{17}$ then $G S(H)=\{\{\sigma(x)=2, \forall x \in V\}\}$.

b) If $(J, \alpha) \in A_{18}$ then $G S(H)=\{\{\sigma(x)=0, \forall x \in V\}\}$.

Proof. a) Consider the configuration $\sigma(x)=2, \forall x \in V$. For any $b \in M$ by (3.3) we have $U\left(\sigma_{b}\right)=U_{17}$. Thus the configuration $\sigma(x)=2, \forall x \in V$ is ground state on the set $A_{17}$.

b) Consider the configuration $\sigma(x)=0, \forall x \in V$. For any $b \in M$ by (3.3) we have $U\left(\sigma_{b}\right)=U_{18}$. Thus the configuration $\sigma(x)=0, \forall x \in V$ is ground state on the set $A_{18}$.

This finishes the proof of Theorem 3.4.

Remark 3.3. 1) Note that if $\sigma(x)=1, \forall x \in V$ is a ground state, then $(J, \alpha) \in A_{16}$, i.e. an external field is equal to zero.

2) In [5] periodic ground states for the Ising model with two step interactions on the Cayley tree and with zero external fields are described. In [6] weakly periodic ground states for the Ising model with competing interactions and with zero external field are described.

So, obviously seen from (Theorem 3.4), when an external field is non-zero translation-invariant, all ground states for the SOS model are translation-invariant.

\section{Model with a periodic external field}

Let $G_{k}^{(2)}=\left\{x \in G_{k}:|x|\right.$ is even $\}$, where $|x|$ means length of the word $x$. Now we shall study $G_{k}^{(2)}-$ periodic ground states for the SOS model with $G_{k}^{(2)}$-periodic external field.

SOS model with $G_{k}^{(2)}$-periodic external field is defined according to the following Hamiltonian:

$$
H(\sigma)=-J \sum_{\langle x, y\rangle \in L}|\sigma(x)-\sigma(y)|+\sum_{x \in V} \alpha_{x} \sigma(x),
$$


where $J, \alpha_{x} \in \mathbb{R}$ and

$$
\alpha_{x}=\left\{\begin{array}{l}
\alpha_{1}, \text { if } x \in G_{k}^{(2)}, \\
\alpha_{2}, \text { if } x \in G_{k} \backslash G_{k}^{(2)},
\end{array}\right.
$$

where $\alpha_{1} \neq \alpha_{2}$ and $G_{k}^{(2)}=\left\{x \in G_{k}:|x|\right.$ is even $\}$.

The energy of a configuration $\sigma_{b}$ on $b$ is defined by the formula

$$
U\left(\sigma_{b}\right)=-\frac{1}{2} J \sum_{x:\left\langle x, c_{b}\right\rangle \in L}\left|\sigma(x)-\sigma\left(c_{b}\right)\right|+\alpha_{c_{b}} \sigma\left(c_{b}\right) .
$$

It is not difficult to prove the following.

Lemma 4.1. We have

$$
U\left(\sigma_{b}\right) \in\left\{U_{1}\left(\sigma_{b}\right), U_{2}\left(\sigma_{b}\right), U_{3}\left(\sigma_{b}\right), U_{4}\left(\sigma_{b}\right), \ldots, U_{29}\left(\sigma_{b}\right)\right\}
$$

for all $\sigma_{b}$. Were

$$
\begin{aligned}
& U_{1}\left(\sigma_{b}\right)=-\frac{J}{2}, \quad U_{2}\left(\sigma_{b}\right)=-J, \quad U_{3}\left(\sigma_{b}\right)=-\frac{3 J}{2}, \quad U_{4}\left(\sigma_{b}\right)=-2 J, \\
& U_{5}\left(\sigma_{b}\right)=-\frac{5 J}{2}, \quad U_{6}\left(\sigma_{b}\right)=-3 J, \quad U_{7}\left(\sigma_{b}\right)=-\frac{J}{2}+\alpha_{1}, \\
& U_{8}\left(\sigma_{b}\right)=-J+\alpha_{1}, \quad U_{9}\left(\sigma_{b}\right)=-\frac{3 J}{2}+\alpha_{1}, \quad U_{10}\left(\sigma_{b}\right)=-\frac{J}{2}+2 \alpha_{1}, \\
& U_{11}\left(\sigma_{b}\right)=-J+2 \alpha_{1}, \quad U_{12}\left(\sigma_{b}\right)=-\frac{3 J}{2}+2 \alpha_{1}, \quad U_{13}\left(\sigma_{b}\right)=-2 J+2 \alpha_{1}, \\
& U_{14}\left(\sigma_{b}\right)=-\frac{5 J}{2}+2 \alpha_{1}, \quad U_{15}\left(\sigma_{b}\right)=-3 J+2 \alpha_{1} \quad U_{16}\left(\sigma_{b}\right)=\alpha_{1}, \\
& U_{17}\left(\sigma_{b}\right)=2 \alpha_{1}, \quad U_{18}\left(\sigma_{b}\right)=0, \quad U_{19}\left(\sigma_{b}\right)=-\frac{J}{2}+\alpha_{2}, \\
& U_{20}\left(\sigma_{b}\right)=-J+\alpha_{2}, \quad U_{21}\left(\sigma_{b}\right)=-\frac{3 J}{2}+\alpha_{2}, \quad U_{22}\left(\sigma_{b}\right)=-\frac{J}{2}+2 \alpha_{2}, \\
& U_{23}\left(\sigma_{b}\right)=-J+2 \alpha_{2}, \quad U_{24}\left(\sigma_{b}\right)=-\frac{3 J}{2}+2 \alpha_{2}, \quad U_{25}\left(\sigma_{b}\right)=-2 J+2 \alpha_{2}, \\
& U_{26}\left(\sigma_{b}\right)=-\frac{5 J}{2}+2 \alpha_{2}, \quad U_{27}\left(\sigma_{b}\right)=-3 J+2 \alpha_{2}, \quad U_{28}\left(\sigma_{b}\right)=\alpha_{2}, \\
& U_{29}\left(\sigma_{b}\right)=2 \alpha_{2} .
\end{aligned}
$$

Definition 4.1. A configuration $\varphi$ is called a ground state of the Hamiltonian (4.1), if

$$
U\left(\varphi_{b}\right)=\min \left\{U_{1}\left(\sigma_{b}\right), U_{2}\left(\sigma_{b}\right), U_{3}\left(\sigma_{b}\right), \ldots, U_{29}\left(\sigma_{b}\right)\right\}
$$

for all $b \in M$.

For a fixed $m=1,2,3, \ldots ., 29$, we set

$$
A_{m}=\left\{\left(J, \alpha_{0}, \alpha_{1}\right) \in \mathbb{R}^{3}: U_{m}=\min \left\{U_{1}\left(\sigma_{b}\right), U_{2}\left(\sigma_{b}\right), U_{3}\left(\sigma_{b}\right), \ldots, U_{29}\left(\sigma_{b}\right)\right\} .\right.
$$

Quite cumbersome but not difficult calculations show that

$$
\begin{aligned}
& A_{1}=A_{2}=A_{3}=A_{4}=A_{5}=\left\{\left(J, \alpha_{0}, \alpha_{1}\right) \in \mathbb{R}^{3}: J=0, \alpha_{1} \geq 0, \alpha_{2} \geq 0\right\}, \\
& A_{6}=\left\{\left(J, \alpha_{0}, \alpha_{1}\right) \in \mathbb{R}^{3}: J \geq 0, \alpha_{1} \geq 0, \alpha_{2} \geq 0\right\}, \\
& A_{7}=A_{8}=A_{9}=\left\{\left(J, \alpha_{0}, \alpha_{1}\right) \in \mathbb{R}^{3}: J=0, \alpha_{1}=0, \alpha_{2} \geq 0\right\}, \\
& A_{10}=\ldots=A_{14}=\left\{\left(J, \alpha_{0}, \alpha_{1}\right) \in \mathbb{R}^{3}: J=0, \alpha_{1} \leq 0, \alpha_{2} \geq \alpha_{1}\right\}, \\
& A_{15}=\left\{\left(J, \alpha_{0}, \alpha_{1}\right) \in \mathbb{R}^{3}: J \geq 0, \alpha_{1} \leq 0, \alpha_{2} \geq \alpha_{1}\right\},
\end{aligned}
$$




$$
\begin{aligned}
& A_{16}=\left\{\left(J, \alpha_{0}, \alpha_{1}\right) \in \mathbb{R}^{3}: J \geq 0, \alpha_{1}=0, \alpha_{2} \leq 0\right\}, \\
& A_{17}=\left\{\left(J, \alpha_{0}, \alpha_{1}\right) \in \mathbb{R}^{3}: J \geq 0, \alpha_{1} \geq 0, \alpha_{2} \leq \alpha_{1}\right\}, \\
& A_{18}=\left\{\left(J, \alpha_{0}, \alpha_{1}\right) \in \mathbb{R}^{3}: J \leq 0, \alpha_{1} \geq 0, \alpha_{2} \geq 0\right\}, \\
& A_{19}=A_{20}=A_{21}=\left\{\left(J, \alpha_{0}, \alpha_{1}\right) \in \mathbb{R}^{3}: J=0, \alpha_{1} \geq 0, \alpha_{2}=0\right\}, \\
& A_{22}=\ldots=A_{26}=\left\{\left(J, \alpha_{0}, \alpha_{1}\right) \in \mathbb{R}^{3}: J=0, \alpha_{1} \geq \alpha_{2}, \alpha_{2} \leq 0\right\}, \\
& A_{27}=\left\{\left(J, \alpha_{0}, \alpha_{1}\right) \in \mathbb{R}^{3}: J \geq 0, \alpha_{1} \geq \alpha_{2}, \alpha_{2} \leq 0\right\}, \\
& A_{28}=\left\{\left(J, \alpha_{0}, \alpha_{1}\right) \in \mathbb{R}^{3}: J \geq 0, \alpha_{1} \leq 0, \alpha_{2}=0\right\}, \\
& A_{29}=\left\{\left(J, \alpha_{0}, \alpha_{1}\right) \in \mathbb{R}^{3}: J \geq 0, \alpha_{1} \leq \alpha_{2}, \alpha_{2} \geq 0\right\} .
\end{aligned}
$$

Theorem 4.1. 1) The following $G_{2}^{(2)}$-periodic configuration

$$
\sigma(x)=\left\{\begin{array}{l}
0, \text { if } x \in G_{2}^{(2)}, \\
1, \text { if } x \in G_{2} \backslash G_{2}^{(2)},
\end{array}\right.
$$

is a ground state on set $\left(J, \alpha_{0}, \alpha_{1}\right) \in A_{3} \cap A_{21}$ for the (4.1) model;

2) The following $G_{2}^{(2)}$-periodic configuration

$$
\sigma(x)=\left\{\begin{array}{l}
1, \text { if } x \in G_{2}^{(2)}, \\
0, \text { if } x \in G_{2} \backslash G_{2}^{(2)},
\end{array}\right.
$$

is a ground state on set $\left(J, \alpha_{0}, \alpha_{1}\right) \in A_{3} \cap A_{9}$ for the (4.1) model;

3) The following $G_{2}^{(2)}$-periodic configuration

$$
\sigma(x)=\left\{\begin{array}{l}
0, \text { if } x \in G_{2}^{(2)}, \\
2, \text { if } x \in G_{2} \backslash G_{2}^{(2)},
\end{array}\right.
$$

is a ground state on set $\left(J, \alpha_{0}, \alpha_{1}\right) \in A_{6} \cap A_{27}$ for the (4.1) model;

4) The following $G_{2}^{(2)}$-periodic configuration

$$
\sigma(x)=\left\{\begin{array}{l}
2, \text { if } x \in G_{2}^{(2)}, \\
0, \text { if } x \in G_{2} \backslash G_{2}^{(2)},
\end{array}\right.
$$

is a ground state on set $\left(J, \alpha_{0}, \alpha_{1}\right) \in A_{15} \cap A_{6}$ for the (4.1) model.

Proof. 1) When we define the configuration for the model of 4.1] on the Cayley tree in the form of (4.3) then $\forall b \in M$ we have $\sigma\left(c_{b}\right)=0$ or $\sigma\left(c_{b}\right)=1$.

If $\sigma\left(c_{b}\right)=0$ then for $\forall x \in S_{1}\left(c_{b}\right)$ we have $\sigma(x)=1$. In this case by 4.2 we take $U\left(\sigma_{b}\right)=U_{3}=\frac{-3}{2} J$.

If $\sigma\left(c_{b}\right)=1$ then $\sigma(x)=0$ for $\forall x \in S_{1}\left(c_{b}\right)$. Then we have $U\left(\sigma_{b}\right)=U_{21}=\frac{-3}{2} J+\alpha_{2}$.

From these cases, $G_{2}^{(2)}$-periodic configuration (see (4.3)) for the model of 4.1) is ground state on the set of $A_{3} \cap A_{21}=\left\{\left(J, \alpha_{0}, \alpha_{1}\right) \in \mathbb{R}^{3}: J=0, \alpha_{1} \geq 0, \alpha_{2}=0\right\}$.

2) If $\sigma\left(c_{b}\right)=1$ then for $\forall x \in S_{1}\left(c_{b}\right)$ we have $\sigma(x)=0$. In this case by 4.2 we take $U\left(\sigma_{b}\right)=U_{9}=$ $\frac{-3}{2} J+\alpha_{1}$.

If $\sigma\left(c_{b}\right)=0$ then $\sigma(x)=1$ for $\forall x \in S_{1}\left(c_{b}\right)$. Then we have $U\left(\sigma_{b}\right)=U_{3}=\frac{-3}{2} J$.

From these cases, $G_{2}^{(2)}$-periodic configuration (see (4.4)) for the model of 4.1 is ground state on the set of $A_{3} \cap A_{9}=\left\{\left(J, \alpha_{0}, \alpha_{1}\right) \in \mathbb{R}^{3}: J=0, \alpha_{1}=0, \alpha_{2} \geq 0\right\}$.

3) If $\sigma\left(c_{b}\right)=0$ then for $\forall x \in S_{1}\left(c_{b}\right)$ we have $\sigma(x)=2$. In this case by 4.2 we take $U\left(\sigma_{b}\right)=U_{6}=-3 J$.

If $\sigma\left(c_{b}\right)=2$ then $\sigma(x)=0$ for $\forall x \in S_{1}\left(c_{b}\right)$. Then we have $U\left(\sigma_{b}\right)=U_{27}=-3 J+2 \alpha_{2}$.

From these cases, $G_{2}^{(2)}$-periodic configuration (see (4.5)) for the model of 4.1) is ground state on the set of $A_{6} \cap A_{27}=\left\{\left(J, \alpha_{0}, \alpha_{1}\right) \in \mathbb{R}^{3}: J \geq 0, \alpha_{1} \geq 0, \alpha_{2}=0\right\}$. 
4) If $\sigma\left(c_{b}\right)=2$ then for $\forall x \in S_{1}\left(c_{b}\right)$ we have $\sigma(x)=0$. In this case by 4.2 we take $U\left(\sigma_{b}\right)=U_{15}=$ $-3 J+2 \alpha_{1}$.

If $\sigma\left(c_{b}\right)=0$ then $\sigma(x)=2$ for $\forall x \in S_{1}\left(c_{b}\right)$. Then we have $U\left(\sigma_{b}\right)=U_{6}=-3 J$.

From these cases, $G_{2}^{(2)}$-periodic configuration (see (4.6)) for the model of 4.1 is ground state on the set of $A_{15} \cap A_{6}=\left\{\left(J, \alpha_{0}, \alpha_{1}\right) \in \mathbb{R}^{3}: J \geq 0, \alpha_{1}=0, \alpha_{2} \geq 0\right\}$.

\section{This finishes the proof of Theorem 4.1.}

Remark 4.1. The configurations

$\sigma^{\prime}(x)=\left\{\begin{array}{l}1, \text { if } x \in G_{2}^{(2)}, \\ 2, \text { if } x \in G_{2} \backslash G_{2}^{(2)}\end{array} \quad\right.$ and $\quad \sigma^{\prime \prime}(x)=\left\{\begin{array}{l}2, \text { if } x \in G_{2}^{(2)}, \\ 1, \text { if } x \in G_{2} \backslash G_{2}^{(2)}\end{array}\right.$

are ground states, if $J=0, \alpha_{1}=0, \alpha_{2}=0$.

Note that in the case $J=0, \alpha_{1}=0, \alpha_{2}=0$ all configurations are ground states.

Acknowledgments. The authors are grateful to Professor U.A.Rozikov for the useful discussions.

\section{References}

1. Rozikov U.A. Gibbs measures on Cayley trees.World scientific.2013.

2. N. N. Ganikhodzhaev, Group representation and automorphisms of the Cayley tree, Dokl. Akad. nauk Resp. Uzbekistan, no. 4, 3 (1994) [in Russian].

3. F.Mukhamedov, Ch.Hee Pah, M.Rahmatullaev, H.Jamil. Periodic and Weakly Periodic Ground States for the $\lambda$ - Model on Cayley Tree. 2017.Journal of Physics: Conf. Series 949, 012021, doi:10.1088/1742 - 6596/949/1/012021.

4. M. I. Kargapolov and Yu. I. Merzlyakov,Fundamentals of the Theory of Groups(Springer-Verlag, New York-Heidelberg-Berlin, 1979). [Fundamentals of Group Theory(Nauka, Moscow, 1982)].

5. U.A.Rozikov. A contructite Description of Grond States and Gibbs Measures for Ising Model with two step interations on Cayley tree. 2006. Journal of statistical Physics. Vol. 122. N2, $217-235$.

6. M. M. Rahmatullaev. Description of Weakly Periodic Ground States of Ising Model with Competing Interactions on Cayley Tree.2010.Applied Mathematics \& Information Sciences 4(2), 237 - 251.

7. M.M.Rakhmatullaev, M.A.Rasulova. Periodic and Weakly Periodic Ground States for the Potts Model with Competing Interactions on the Cayley Tree.2016. ISSN 1055-1344, Siberian Advances in Mathematics, Vol. 26, No.3, pp.215 - 229.

8. Rozikov U. A., Rahmatullaev M. M. Weakly Periodic Ground States and Gibbs Measures for the Ising Model with Competing Interactions on the Cayley Tree, Theor. Math. Phys. 160, No. 3, 1292-1300 (2009).

9. Rahmatullaev M. M., Rasulova M. A. Ground States for the Ising model with an external field on the Cayley tree, Uz. Math. Journal, No. 3, 147-155 (2018).

10. Rozikov U. A., Description of limiting Gibbs measures for $\lambda$-models on the Bethe lattice. Sib.Math.J. 39(1998) 427-435.

11. Rozikov U. A., Describing uncountable number of Gibbs measures for inhomogeneous Ising model, Theor. Math. Phys. 118 (1999) 95-104.

12. Ganikhodjaev N. N. and Rozikov U. A., Description of periodic extreme Gibbs measures of some lattice models on the Cayley tree, Theor. Math. Phys. 111 (1997) 480-486. 
13. Ganikhodjaev N. N. and Rozikov U. A., On disordered phase in the ferromagnetic Potts model on the Bethe lattice, Osaka J. Math. 37 (2000) 373-383.

14. Rozikov U. A., Suhov Y. M., Gibbs measures for SOS models on a Cayley tree, Infinite Dimensional Analysis, Quantum Probability and Related Topics, Vol. 9, No. 3 (2006) 471-488.

15. Mazel A. E. and Suhov Yu. M., Random surfaces with two-sided constraints: An application of the theory of dominant ground states, J. Statist. Phys. 64 (1991) 111-134.

Rahmatullaev M.M.

Institute of mathematics, Tashkent, Uzbekistan; Namangan State Universite, Namangan, Uzbekistan, e-mail: mrahmatullaev@rambler.ru

Abdusalomova M. R.

Namangan State Universite, Namangan, Uzbekistan, email: mahliyo13abdusalomova@gmail.com

Rasulova M.A.

The Ministry of International Affairs Namangan academic lyceum, Namangan, Uzbekistan, e-mail: m_rasulova_a@rambler.ru 